\title{
Prácticas pedagógicas de la enseñanza y el aprendizaje, declarado en el modelo educativo pedagógico de la universidad Santo Tomás, Colombia.
}

\section{Pedagogical practices of teaching and learning, approach, declared in the educational pedagogical model of the Santo Tomás university, Colombia.}

\author{
Iliana Fernández Peña ${ }^{1}$, Fabiola Inés Hernández Barriga², Lina María Fonseca Ortiz³ , Iván René León \\ Garzón ${ }^{4}$, Wilson Arley Sánchez Pinzón ${ }^{5}$
}

1Universidad Santo Tomás, Colombia, ilianafernandez@usantotomas.edu.co, 2Universidad Santo Tomás, fabiolahernandez@usantotomas.edu.co, 3Universidad Santo Tomás, linafonseca@usantotomas.edu.co, ${ }^{4}$ Universidad Santo Tomás, ivanleon@usantotomas.edu.co, ${ }^{5}$ Universidad Santo Tomás, wilson.sanchez@usantotomas.edu.co

\section{RESUMEN}

La Universidad Santo Tomás (USTA), a través de su Modelo Educativo Pedagógico (MEP), señala una clara opción por la pedagogía problémica y la metodología problematizadora del saber, lo cual motiva a que sus principales actores, docentes y estudiantes, realicen una delimitación de las problemáticas sobre las que la academia debe enfocarse en la búsqueda de soluciones y alternativas para contribuir a las necesidades de la sociedad y del país. Desde este panorama, las debilidades del quehacer docente, influenciadas por las visiones que asume frente a la enseñanza y el aprendizaje, limitan las acciones tendientes al desarrollo de las competencias de los estudiantes en diversos ambientes académicos. Problema científico: ¿Qué prácticas pedagógicas, circunscritas al enfoque problémico, declarado en el MEP de la USTA, prevalecen en la muestra objeto de estudio en relación con los procesos de enseñanza y aprendizaje en los programas de pregrado de la Sede Principal?, objetivo: Caracterizar los procesos de enseñanza y aprendizaje, en coherencia con el enfoque problémico que prevalecen en la muestra objeto de estudio de los programas de pregrado de la USTA - Sede Principal. Se realizó revisión documental de concreciones curriculares de la muestra, se aplicó encuesta y grupo focal a miembros del Comité Particular de Currículo y Docencia y encuesta en línea a 493 docentes. Resultados: insuficiente uso de estrategias didácticas acorde con el enfoque problémico, los ritmos y estilos de aprendizaje que propicien el aprendizaje autónomo, participativo, colaborativo y el desarrollo de competencias y del pensamiento crítico y creativo.

Palabras clave: prácticas pedagógicas; enseñanza y aprendizaje; enfoque problémico. 


\section{ABSTRACT}

The Universidad Santo Tomas (USTA), through its Educational Pedagogical Model (MEP), points out a clear option for problem-solving pedagogy and problematizing methodology of knowledge, which motivates its main actors, teachers and students, to carry out a delimitation of the problems on which the academy should focus on finding solutions and alternatives to contribute to the needs of society and the country. From this perspective, the weaknesses of the teaching task, influenced by the visions that it assumes in front of the teaching and learning, limit the actions tending to the development of the competences of the students in diverse academic environments. Scientific problem: What pedagogical practices, circumscribed to the problémico approach, declared in the MEP of the USTA, prevail in the sample object of study in relation to the teaching and learning processes in the undergraduate programs of the Headquarters?, objective: Characterize the teaching and learning processes, in coherence with the problémico approach that prevails in the sample object of study of the undergraduate programs of the USTA - Main Headquarters. A documentary review of the curricular concretions of the sample was carried out, an online survey was applied to 493 teachers, a survey and a focus group for members of the Curriculum and Teaching Particular Committee. Results: insufficient use of didactic strategies according to the problémico approach, the rhythms and styles of learning that propitiate the autonomous, participative, collaborative learning and the development of competences and of the critical and creative thought.

Keywords: pedagogical practices; teaching and learning; problémico approach.

\section{INTRODUCCIÓN}

En la actualidad la educación superior se ve influenciada por multiplicidad de fenómenos contextuales que la tensionan de manera permanente y le exigen responder asertivamente a estas transformaciones. Algunos de los retos asumidos son: ampliación de la cobertura, mejoramiento de los sistemas de control y vigilancia de la calidad de la educación superior, inclusión, articulación de las funciones sustantivas, entre otros. De acuerdo con el Centro Interuniversitario de Desarrollo (2016) "La política pública en materia de educación superior ha estado centrada desde años atrás (Decreto 80 de 1980) en los denominados problemas crónicos de la educación superior tanto pública como privada a saber: mejoramiento del acceso; búsqueda de un incremento significativo de calidad; mejor pertinencia de la oferta educativa de tercer nivel; el logro de una mayor equidad; la sostenibilidad financiera y la eficiencia del sistema de aseguramiento de la calidad". (Centro Interuniversitario de Desarrollo (CINDA), 2016, pág. 227). 
Para el caso del ámbito institucional específico de la Universidad Santo Tomás, el Modelo Educativo Pedagógico (MEP), se encuentra estrechamente relacionado con el Proyecto Educativo Institucional (PEI), el cual es que el que determina los componentes del modelo, en tanto lo ubica contextualmente de acuerdo con las necesidades, expectativas y características de formación propias de la Universidad. De hecho, es el mismo Proyecto educativo Institucional (PEI) que, en relación con el modelo, enuncia la misión, la historia, los objetivos estatutarios, la formación integral y el currículo, las funciones sustantivas y la interacción con el medio externo (USTA, PEI, 2010, pág. 10).

Con base en el Modelo Educativo Pedagógico Institucional, estos criterios de organización de las prácticas de enseñanza y aprendizaje, de la investigación y de los procesos de responsabilidad social están caracterizados por ser problémicos, es decir, el Modelo Pedagógico Institucional opta por la pedagogía problémica y la metodología problematizadora. Entendiendo que la primera posee unos antecedentes históricos del pensamiento tomista y cuyo método propone partir de problemas enunciados claramente, su división en subproblemas y temas que llevan a la investigación de un desarrollo sistémico y lógico que enfatiza en las opiniones contrarias y finalmente, en una toma de postura resolutiva frente al problema central (USTA, Modelo Educativo Pedagógico, 2010, pág. 62).

Por su parte, la metodología problematizadora hace referencia a la construcción de preguntas de una realidad que se presenta de manera problemática. Entonces, lo problémico se materializa en el abordaje pedagógico e implica la mirada de las prácticas de enseñanza y aprendizaje de un proceso focalizado. Es decir, este tipo de modelo lo que propone es que tanto docentes como estudiantes se pongan en disposición de una actitud de diálogo inter y transdisciplinar que permita la discusión y posterior construcción de unas nuevas realidades.

Respecto al proceso de enseñanza y aprendizaje, la Universidad Santo Tomás a través de su Modelo Educativo - Pedagógico, presenta "la perspectiva de Tomas de Aquino y de la tradición pedagógica dominicana, el rol y la función del maestro no puede ser unilateral y autoritaria, ni la actividad del alumno puede concebirse como un activismo protagónico en solitario, sino que juntos entran en un proceso constructivo y amigable de dialogo e interacción colaborativa y respetuosa con respecto al aprendizaje y la adquisición del saber". (Modelo y Pedagógico USTA, 2010, p. 52). Así, el Modelo Educativo Pedagógico señala unas características que deben ser consideradas y contenidas en la relación enseñanza y aprendizaje, profesor y estudiante, por esta razón, las competencias a desarrollar en el profesional tomasino se encuentran fundamentadas en las dimensiones de la acción humana formuladas por Santo Tomás y explícitas en la Política Curricular (2004) a saber: comprender, obrar, hacer y comunicar y responden al propósito de la Universidad al "Formar líderes con sentido crítico de la realidad y compromiso ético para llevar a cabo los cambios necesarios en la vida social y promover el desarrollo integral de nuestro pueblo" (p.59) 
Desde este panorama, las debilidades del quehacer docente, influenciadas por las visiones que asume frente a la enseñanza y el aprendizaje, limitan las acciones tendientes al desarrollo de las competencias de los estudiantes en diversos ambientes académicos, por ello, la Unidad de Desarrollo Curricular y Formación Docente (UDCFD), adscrita a la Vicerrectoría Académica General, en articulación con la Facultad de Comunicación, desarrolla un proyecto de investigación, el cual se ha estructurado en etapas y una de ellas pretende dar respuesta al siguiente problema científico: ¿Qué prácticas pedagógicas, circunscritas al enfoque problémico, declarado en el MEP de la USTA, prevalecen en la muestra objeto de estudio en relación con los procesos de enseñanza y aprendizaje en los programas de pregrado de la Sede Principal?, objetivo: Caracterizar los procesos de enseñanza y aprendizaje, en coherencia con el enfoque problémico que prevalecen en la muestra objeto de estudio de los programas de pregrado de la USTA - Sede Principal.

\section{METODOLOGÍA}

Para la ejecución del proyecto de investigación, este se dividió en tres etapas. En este artículo, se muestra el cómo se procedió para desarrollar la primera etapa. Se realizó un estudio descriptivo, que busca el análisis de la coherencia curricular a nivel USTA (Sede principal) y los diferentes programas con respecto a las prácticas didácticas, a partir de las siguientes categorías: Modelo Educativo Pedagógico (MEP), coherencia entre las concreciones curriculares (Proyecto Educativo Programa (PEP), Plan Analítico del Programa (PAP) y Syllabus, prácticas de enseñanza y aprendizaje (estrategias didácticas y evaluativas), estilos de enseñanza, ritmos de aprendizaje, fortalezas y debilidades de las prácticas de enseñanza y aprendizaje, didácticas específicas.

A partir de un muestreo estratificado se seleccionó la muestra de los syllabus a valorar. En este caso, los estratos son la cantidad de programas (50) con un total de 3053 syllabus, 50 PEP y 50 PAP. Se consideró analizar el $25 \%$ de los syllabus, por lo que se valoraron 763 , luego, fue necesario distribuir el tamaño de esta muestra (763) de forma proporcional a través del total de estratos (50). En relación con los PEP y PAP, se analizó el total en cada programa.

Sobre la base de esta información se identificaron la cantidad de syllabus a analizar para cada programa (estrato), a partir de una asignación proporcional. Posteriormente, a los syllabus de la muestra y a los PEP y PAP de cada programa, se les realizó una revisión documental, teniendo en cuenta las matrices elaboradas por el área de currículo de la Unidad de Desarrollo Curricular y Formación Docente (UDCFD), que permitió orientar el análisis de las concreciones curriculares.

Posteriormente, se aplicó una encuesta inicial a los líderes del Comité Particular de Currículo y Docencia (CPCD), con el propósito de determinar una aproximación a las percepciones y maneras de cómo se están abordando las prácticas pedagógicas desde los procesos de enseñanza y aprendizaje, en las 
facultades, departamentos e Instituto de Lenguas Fray Bernardo de Lugo, se desarrolló además un Grupo Focal con dichos líderes donde se reflexionaron tópicos sobre las prácticas de enseñanza exitosas en el ejercicio docente, las razones de porqué las consideran exitosas y los resultados de aprendizajes obtenidos en los estudiantes.

Se aplicaron además, 493 encuestas on-line a docentes de pregrado de los programas académicos vigentes y activos de este nivel de formación (Sede Principal). Para el análisis de las mismas se utilizó la herramienta de Minería de Textos que busca extraer información útil e importante de formatos de documentos heterogéneos, en este caso se utilizó para analizar las respuestas a las preguntas abiertas realizadas en la encuesta online, a través de determinados pasos (Procesamiento de la Información, limpieza del texto, lematización, análisis, tabla léxica y nube de palabras), la herramienta posibilitó obtener información que permitió generar análisis sobre las respuestas de los profesores. De este modo, se analizaron los resultados obtenidos a nivel institucional que permitieron caracterizar las prácticas en el ejercicio áulico en relación con las orientaciones curriculares a nivel USTA.

\section{RESULTADOS Y DISCUSIÓN}

Como parte del proyecto de investigación, en este artículo se muestran resultados obtenidos de la primera etapa. Se partió de la revisión documental de matrices elaboradas por el área de currículo de la Unidad de Desarrollo Curricular y Formación Docente (UDCFD), que permitió orientar el análisis de la coherencia entre las concreciones curriculares y la obtención de los siguientes resultados:

Los programas que forman parte de la muestra objeto de estudio responden a la política curricular de la pedagogía problémica, lo cual constituye una apuesta propia de la Universidad Santo Tomás, en la que el currículo toma como punto eje de la formación cuestionamientos derivados de las realidades cotidianas, que se ponen en diálogo con las perspectivas y los sustentos teóricos de las diferentes disciplinas.

La comprensión de Santo Tomás de Aquino en su método prudencial y educativo, facilita leer críticamente la realidad que es problematizadora, desde un pensamiento problémico que no fragmenta el conocimiento en las disciplinas, sino que permite abordarlo de manera transdisciplinar a través de los problemas y su estudio.

El Proyecto Educativo Pedagógico (PEP), es un documento o concreción curricular que se constituye en ruta de navegación de un programa académico, pues esboza principios, orientaciones y políticas que dirigen su desarrollo. Declara, entre otros, la misión, la visión y el propósito de formación que le da identidad (UDCFD-USTA, 2015). En cada programa analizado, como proyecto curricular único, se plasman las intencionalidades educativas, pedagógicas, curriculares y didácticas, la forma de relacionarse con el entorno, así como la visibilización de la interrelación de las funciones sustantivas en 
el programa académico, por lo que se identifica coherencia y pertinencia con la filosofía y lineamientos institucionales plasmados en el Proyecto Educativo Institucional (PEI), se expresan las necesidades del contexto y la dinámica particular de cada Programa Académico.

A través del análisis de la revisión de los PAP, "documento o concreción curricular que permite planear semestralmente el desarrollo asertivo, pertinente y oportuno de los programas académicos, se constituye en la bitácora de estrategias académicas, mecanismos y acciones concretas iluminadas por el PEP" (UDCFD-USTA, 2015), permitió reconocer articulación epistemológica con el PEP de cada programa y se identificaron las siguientes regularidades en las que hay que consolidar su fortalecimiento tales como: profundizar en la pertinencia del programa en contexto internacional, ya que enfocan el análisis fundamentalmente a los contextos local e institucional, descripción pedagógica a la luz de los propósitos del programa y las estrategias a desarrollar en el proceso de formación (Proyección Social Universitaria, formación investigativa e investigativa formativa), estrategias didácticas y evaluativas que el programa aplica o promueve en coherencia con el marco pedagógico institucional, identificar los espacios académicos, que de manera intencional, fomentan las competencias en investigación y cuáles propician la identificación de problemas de la(s) disciplina(s) y el diseño de alternativas de solución a los mismos, enunciar y describir las estrategias de Proyección Social que desarrolla el programa en el marco de las seis institucionales (desarrollo comunitario, egresados, educación continua, asesorías y consultorías, emprendimiento, internacionalización y relaciones interinstitucionales - convenios vigentes y en ejecución), expresar de manera concreta la interdisciplinariedad en el manejo de los espacios académicos, referirse a los aportes de otras disciplinas en la solución de los problemas propios y objeto de estudio de cada programa, así como determinar la forma de desarrollo, acentuar en el perfil del egresado, los rasgos del egresado tomasino, asimismo, lograr más articulación con el enfoque curricular del programa declarado en el PEP.

Al analizar los syllabus de la muestra de estudio como concreción curricular se identifica un primer acercamiento a la práctica pedagógica del ejercicio áulico, y se encuentran precisiones en las cuales hay que profundizar, tales como: expresión de manera concreta de los propósitos u objetivos que se persiguen con cada espacio académico para la formación integral del profesional en formación, en coherencia con el perfil del egreso declarado en el PEP de cada programa, determinación de las competencias a desarrollar en los estudiantes en articulación con las dimensiones de la acción humana como política curricular, concreción de los resultados de aprendizajes orientados al logro de las competencias, ajustar las estrategias didácticas y actividades en función de obtener los resultados de aprendizajes, que contribuyen al desarrollo de las competencias, ahondar en la coherencia entre competencia, resultado de aprendizaje, estrategia didáctica, actividades y estrategia de evaluación, uso de estrategias didácticas mediadas por la Tecnología de la Informática y las Comunicaciones (TIC), 
intencionalidad en el uso de segunda lengua como competencia transversal, sistematización del uso de rúbricas.

Como parte de estos resultados y en coherencia con la política curricular se crea el Sistema Institucional de Evaluación de los Aprendizajes -SEA-. apuesta institucional liderada por la Vicerrectoría Académica General, a través de la Unidad de Desarrollo Curricular y Formación Docente, que se constituye en insumo para todas las facultades, en sus diferentes niveles de formación y modalidades, Departamentos Académicos e Instituto de Lenguas, para que desde los ejercicios propios de la reflexión pedagógica, articulada y consensuada de los diversos colectivos docentes, se actualice el formato syllabus, de acuerdo con los aspectos abordados en el SEA: competencias, resultados de aprendizaje, estrategias didácticas y evaluativas, rúbricas de evaluación y recursos.
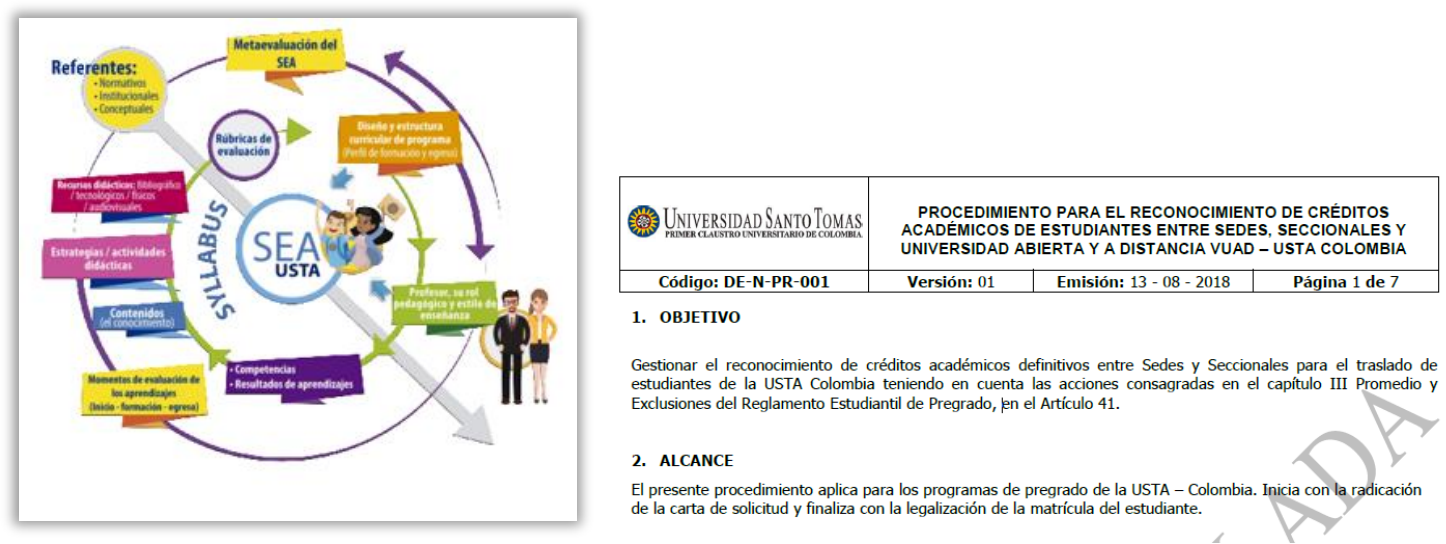

Fuente: Unidad de Desarrollo Curricular y Formación Docente (UDCFD, 2018)

\section{Resultados Encuesta Inicial Aplicada a Miembros del Comité Particular de Currículo y Docencia}

De manera general los miembros del CPCD, en el semestre 2017, no poseen una conceptualización clara de lo que constituyen las estrategias didácticas, las que presuponen el cómo se enseña y cómo aprende el alumno, existe confusión en cuanto a estrategias, métodos, técnicas, actividades, medios de enseñanza, lo que puede ser atribuido a los múltiples criterios que se ofrecen desde las Ciencias Pedagógicas al ser conceptualizados, por ello la necesidad de contar con una precisión y asunción teórica.

En este sentido, el autor Ortiz, 2004, expresa que la estrategia didáctica, presupone enfocar el cómo enseña el docente y cómo aprende el alumno a través de un proceso donde los últimos aprendan a pensar, a participar activa, reflexiva y creadoramente. En tal sentido, las estrategias didácticas no se limitan a los métodos y formas con los que se enseña, sino que además incluyen acciones que tienen en cuenta el repertorio de procedimientos, técnicas y habilidades que tienen los estudiantes para aprender, 
lo cual como expresa este autor, es una concepción más consecuente con las tendencias actuales de la Didáctica.

Sin embargo, en la práctica no siempre resulta fácil delimitar claramente las fronteras que separan las estrategias docentes de los métodos de enseñanza aprendizaje, ni de estos con las técnicas y procedimientos que componen un método; por ejemplo, algunos prefieren utilizar el término estrategia de enseñanza-aprendizaje por el de métodos de enseñanza-aprendizaje, otros lo usan indistintamente (...) (Montes de Oca, Machado Ramírez, 2011)

En cuanto a los criterios referidos a las categorías de estilos de enseñanza y ritmos de aprendizaje, los encuestados refieren que "el estilo de enseñanza debe estar orientado a la metodología problémica en coherencia con la formación integral de los estudiantes" , expresan que "el docente debe conocer, a partir del diagnóstico de aprendizaje, los diferentes ritmos que poseen sus estudiantes para aprender, y que sobre la base de esa caracterización, debe planear sus estrategias didácticas y tareas a desarrollar por los estudiantes en función del logro de los resultados de aprendizajes y las competencias". Se considera necesario facilitar a los alumnos un direccionamiento organizado e información estructurada, en otras ocasiones, proporcionales modelos de acción a incorporar, indicaciones y sugerencias detalladas, y en otras, hacer que elijan y desarrollen en forma autónoma las actividades de aprendizaje. En este sentido, se comparte con los autores (Ortiz y Aguilera, 2005) cuando refieren que al ser el proceso de enseñanza y aprendizaje dirigido, los estilos de aprendizajes se encuentran condicionados por la enseñanza y específicamente por las estrategias de enseñanza que desarrollan los profesores.

Sobre la Didáctica Específica, entendida como aquellas que puedan ser desarrolladas y adaptadas al contexto de los educandos(...) (León, 2017), de manera general, los encuestados afirman que están fundamentadas en las particularidades de los procesos de enseñanza y aprendizaje y que es el docente a través de sus competencias pedagógicas y didácticas, quien las aplica de manera exitosa o no en el proceso formativo, sin embargo, consideran que estas se aplican desde lo disciplinar y que la manera de llevarla al ejercicio áulico, tiene que ver con los referentes a través de los cuales se formaron, independientemente de la actualización sistemática que reciben mediante la cualificación.

Dentro de sus fortalezas como docente reconocen: "conocimiento del tema, experiencia docente y profesional, seguimiento a estudiantes, respeto y buenas relaciones con los estudiantes, gusto por la lectura, comprensión del humanismo, procesos particulares con los estudiantes, proyecto integrador, metodología participativa, diversidad de estrategias, problematización, planeación" y como debilidades: "falta de conocimiento de herramientas tecnológicas, no se sistematizan las experiencias, insuficiente uso del Moodle, poco tiempo para retroalimentar, muchas clases teóricas, no se tiene en cuenta la realidad para construir conocimiento, debilidad en lengua extranjera, falta de estrategias para motivar más a los estudiantes, fortalecer la problematización del saber, poco seguimiento al trabajo en plataformas". 


\section{Resultados de la aplicación del grupo focal a miembros del CPCD}

El $65 \%$ de los miembros del grupo focal consideran las siguientes prácticas de enseñanza como exitosas en su ejercicio docente: Aprendizaje Basasdo en Problemas, Aprendizaje Basado en Proyectos y Estudio de caso, Storytelling, mientras que el 35\% relacionan acciones, actividades, ejercicios, uso de la plataforma Moodle, guìs de trabajo, talleres, clase magistral y trabajo colaborativo, dentro de las razones expuestas del porqué las prácticas de enseñanza que refiren son exitosas están: " favorecen el trabajo autónomo, el protagonismo, la cooperación, libertad de expresión dentro del respeto, desarrollo de compentencia, desarrollo del pensamiento crítico, socialización de expreiencias, trabajo individual, grupal, uso del aula virtual", entre otras.

\section{Resultados de la aplicación de encuesta on-line a 493 docentes de pregrado de los programas académicos vigentes y activos de este nivel de formación (Sede Principal) de la USTA.}

Ante la pregunta, describa máximo tres estrategias que Usted generaría en el aula para facilitar el aprendizaje de todos los estudiantes teniendo en cuenta sus diferencias, se realizó el análisis de las mismas mediante la utilización de la herramienta de Minería de Textos.

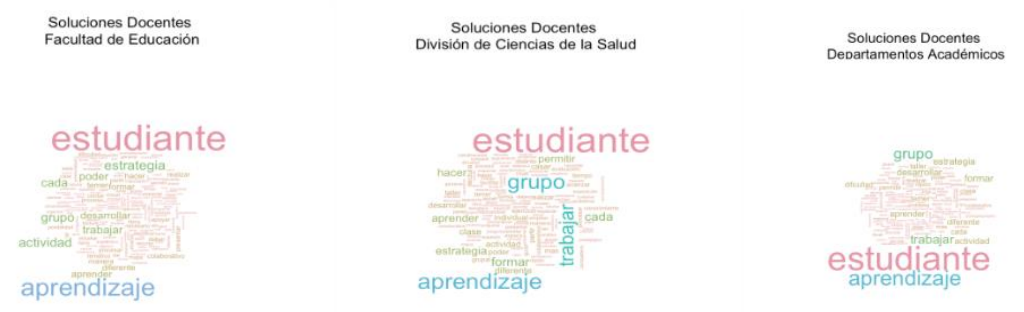

Fuente: ejemplos de nubes de palabras resultantes de las tablas léxicas

De manera general como se aprecia en las nubes de palabras, a partir de las respuestas ofrecidas por los docentes encuestados on-line, en el $100 \%$ de las respuestas se manifiesta con mayor frecuencia la palabra estudiante, como centro del proceso de enseñanza y aprendizaje, asimismo le continúan: aprendizaje, grupo, estrategia, formar, hacer, habilidad, colaborativo, actividad, aula virtual, ritmos de aprendizaje, talleres, solución de problemas, por lo que se interpreta que para contribuir al desarrollo de la formación integral de los estudiantes, se utilizan estrategias en los diferentes espacios académicos, que conllevan al trabajo grupal, colaborativo, mediante actividades, talleres, dirigidas al desarrollo de competencias, al hacer, como una de las dimensiones de la acción humana declarada en el MEP, uso del aula virtual, mediante el acompañamiento, la tutoría, teniendo en cuenta los ritmos de aprendizaje, la 
individualidad, apoyándose en materiales de enseñanza, la motivación, y con la finalidad de dar solución a los problemas contextuales.

\section{CONCLUSIONES}

1. La revisión documental de las concreciones curriculares de la muestra objeto de estudio permitió identificar que existe articulación entre ellas, sin embargo, en los syllabus, debe fortalecerse las intencionalidades epistemológicas declaradas en los PEP y PAP de cada programa en coherencia con el enfoque problémico, expresado en el Modelo Educativo Institucional.

2. La interpretación y el análisis de los resultados, permite concluir que a pesar de que existen Lineamientos para el diseño y la actualización curricular, en la práctica áulica persisten diversidad de interpretaciones en relación con la utilización de estrategias didácticas y evaluativas, falta de claridad en la definición de las competencias, fundamentadas en las dimensiones de la acción humana formuladas por Santo Tomás y explícitas en la Política Curricular, de igual manera los resultados de aprendizajes. Los docentes expresan claridad en sus consideraciones sobre las didácticas específicas, estilos de enseñanza y ritmos de aprendizaje.

\section{REFERENCIAS BIBLIOGRÁFICAS}

Centro Interuniversitario de Desarrollo (CINDA). (2016). Educación superior en Iberoamérica. Santiago de Chile: RIL® editores.

Klauss, A (2017). Didáctica, didáctica general, didácticas específicas y metódicas: tensiones productivas

y determinación del punto de observación. Recuperado de http://web.usbmed.edu.co/usbmed/CURSO DOCENTE/PORTAFOLIO6/G6CAUCASIA IDAMITH LEON INFORMES CURRICULO.pdf

Montes de Oca, N. y Machado, E. (2011). Estrategias docentes y métodos de enseñanza-aprendizaje en la Educación Superior. Recuperado de https://www.researchgate.net/publication/260771008

Ortiz, E. y Mariño, M. (2004 ). Estrategias educativas y didácticas en la educación superior. Tendencias actuales de la didáctica en la educación superior, 9 (5), s/p.

Ortiz, E. y Aguilera, E. (2005).Los estilos de aprendizajes de los estudiantes universitarios y sus implicaciones didácticas en la Educación Superior, 10 (5), 1-9.

USTA. (2004). Política Curricular. Política. Bogotá, Colombia: USTA.

USTA. (2010). Proyecto Educativo Institucional. Bogotá: USTA.

USTA. (2010). Modelo Educativo Pedagógico. Bogotá, Colombia: USTA.

USTA. (2010). Proyecto Educativo Pedagógico, Colombia: USTA. 